\title{
Coherencia entre el currículum formal y currículum real del curso Psicología en la carrera Médico y Cirujano
}

\section{Coherence between the formal curriculum and the actual curriculum of the Psychology course in the Medical and Surgeon career}

\section{Referencia}

\author{
Vilma Estela García Marroquín \\ Maestría en Currículum \\ Facultad de Humanidades \\ Universidad de San Carlos de Guatemala \\ vilmagarcia21@gmail.com
}

García Marroquín, V. E. (2021). Coherencia entre el currículum formal y currículum real del curso Psicología en la carrera Médico y Cirujano. Revista Guatemalteca de Educación Superior, 4(1), 1-11. DOI: https://doi.org/10.46954/revistages.v4i1.48

Recibido: 15/11/2020

Aceptado: 16/01/2021

\section{Resumen}

El artículo presenta los resultados de la evaluación curricular, realizada en la unidad didáctica de psicología, de la facultad de ciencias médicas, de la Universidad de San Carlos de Guatemala, se realizó con el objetivo de determinar la coherencia que existe entre el currículum formal y el currículum real de dicha unidad didáctica.

El estudio tuvo un enfoque mixto, ya que por su naturaleza y los sujetos a investigar era importante conocer su perspectiva tanto subjetiva como objetiva, por lo que se eligió técnicas de recolección de datos tanto cualitativos como cuantitativos, con un diseño exploratorio secuencial, de tipo descriptivo. La 
recopilación de información fue basada en dos cuestionarios, el primero aplicado a 80 estudiantes de tercero y sexto año de la carrera de médico y cirujano, el segundo se aplicó a 25 médicos egresados. Se realizó un grupo focal, con 9 docentes, que integran el claustro de profesionales que imparten la asignatura de psicología a los estudiantes del primer año de la carrera de medicina.

Los principales resultados fueron los siguientes: el $43 \%$ de los estudiantes opinó que muchas veces el contenido del programa del curso fue afín con los temas recibidos en clase. El $41.3 \%$ opinó que siempre se cumplió con lo establecido en el programa. Mientras que el $33.3 \%$ de los egresados consideró que el aporte del curso de psicología en su carrera como médicos, está relacionado con la conceptualización de la materia. El 25\% respondió que el curso les aportó contenido relacionado con la integración de la conceptualización, aplicación, reflexión, darle un sentido a la vida cotidiana y las competencias laborales que el médico necesita desempeñar.

En cuanto a la perspectiva y el paradigma educativo, existe una discrepancia entre estudiantes y egresados, ya que el $43.8 \%$ de los estudiantes, opinan que el currículum de psicología aplica una perspectiva critica o de evaluación para la acción y el $56.3 \%$ considera que el paradigma educativo es crítico, mientras que el $47.8 \%$ de los egresados, considera que la perspectiva que se utiliza en el currículum de psicología es hermenéutico o de la evaluación para la comprensión y el 37.5\% ubica el currículum en un paradigma cuantitativo.

Palabras clave: currículum, unidad didáctica, paradigmas, perspectivas, competencias.
Es necesario hacer una reformulación del currículo. Es por ello, por lo que se hacen las recomendaciones respectivas para dar solución a esta problemática y de esta manera se hagan los ajustes necesarios al currículo de la unidad evaluada. 


\section{Abstract}

This article presents the results of the curricular evaluation, made at the didactic unit of psychology, of the faculty of medical sciences, of the University of San Carlos de Guatemala, it was carried out with the objective of the need to determine the coherence that exists between the formal curriculum and the actual curriculum.

The study had a mixed approach, with a descriptive sequential exploratory design, the collection of information was based on two questionnaires, the first applied to 80 students in the third and sixth years of the medical and surgeon career, the second was applied to 25 graduated doctors. A focus group was held, with 9 teachers, who make up the faculty of professionals who teach the subject of psychology to students of the first year of the medical career.

The main results were, $43 \%$ of the students thought that many times the content of the course program was similar to the topics received in class. $41.3 \%$ believed that the provisions of the program were always met. While $33.3 \%$ of the graduates considered that the contribution of the psychology course in their career as doctors is related to the conceptualization of the subject. $25 \%$ responded that the course provided them with content related to the integration of conceptualization, application, reflection, giving meaning to daily life and the job skills that the doctor needs to perform.

Regarding the educational perspective and paradigm, there is a discrepancy between students and graduates, since $43.8 \%$ of the students believe that the psychology curriculum applies a critical or evaluation perspective for action and $56.3 \%$ consider that the educational paradigm is critical, while $47.8 \%$ of the graduates consider that the perspective used in the psychology curriculum is hermeneutical or that of evaluation for understanding, and $37.5 \%$ places the curriculum in a quantitative paradigm.

It is necessary to reformulate the curriculum. That is why the respective recommendations are made to solve this problem and in this way make the necessary adjustments to the curriculum of the evaluated unit.
Keywords: curriculum, didactic unit, paradigms, perspectives, competences. 


\section{Introducción}

El currículum como un plan que coadyuva al aprendizaje es una guía de planificación que influye en la toma de decisiones, específicamente en tres aspectos importantes: 1) contenido; 2) experiencias de aprendizaje; 3) planes para llevar a cabo aprendizaje, según Taba (citada por Casanova, 2009, p.87).

Los cambios que enfrenta la educación han puesto al currículum como parte fundamental de los programas educativos, ya que nos da parámetros de la metodología a utilizar, didáctica y estrategias que los docentes pueden implementar para lograr una formación integral.

La evaluación curricular comprende la recopilación de información de un programa educativo, contextualizando su pertinencia, necesidades y problemáticas a mejorar. Este proceso nos brinda un diagnóstico de la realidad, por medio de la compilación de información tanto interna como externa del programa, haciendo una valoración de las evidencias obtenidas, identificando aspectos a intervenir y haciendo de este proceso algo permanente y sistemático que coadyuve a la mejora continua.

La investigación realizada fue conveniente académicamente en la facultad de ciencias médicas, por la necesidad de diseñar un plan de mejora que se pueda implementar en la práctica médica y así mejorar en proceso de enseñanza aprendizaje.

Es importante una concordancia entre los diversos tipos de currículum (formal, real y oculto), en la investigación realizada, se tomó en cuenta el currículum formal, el cual nos ayuda a planificar todo el proceso de enseñanza aprendizaje, con la finalidad de que exista una fundamentación en la puesta en práctica de lo planteado en la programación y lo que se evidencia en el currículum real, el cual describe la realidad en el aula (Casarini,1999). 
Es de relevancia social ya que la Universidad de San Carlos de Guatemala, siendo una universidad estatal, egresa la mayor cantidad de médicos en el país, un egresado no solo con conocimientos en medicina, sino también con una adecuada salud mental, brindará un servicio social - comunitario adecuado para mejorar la perspectiva y labor que se tiene de la salud publica en el país.

Con esta evaluación, basada en la programación didáctica, perspectiva y paradigmas educativos, contenido, metodología y evaluación, se puede obtener información para la toma de decisiones, tales como: la actualización de contenido, actualización o rediseño curricular, replantear el programa académico. Por esto la importancia de que la evaluación curricular sea un proceso sistemático y permanente.

Con este artículo se pretende dar a conocer los resultados de los datos recopilados en la investigación, y dar respuesta a los objetivos planteados, determinando la coherencia existente entre el currículum formal y el currículum real de la unidad didáctica de psicología, de la facultad de ciencias médicas, de la Universidad de San Carlos de Guatemala, identificar la perspectiva, paradigma y modelo educativo, describir aspectos a intervenir en el programa y dar a conocer las recomendaciones que se realizaron al concluir la investigación.

\section{Materiales y Métodos}

Para la recopilación de la información, se trabajó con 3 tipos de informantes importantes en el currículum de la unidad didáctica de psicología, de la faculta de ciencias médicas, de la Universidad de San Carlos de Guatemala, a) estudiantes, b) egresados y c) docentes. Para ello se utilizaron cuestionarios virtuales aplicados a los primeros 2 sujetos antes mencionados y se realizó un grupo focal con los docentes de la unidad didáctica evaluada. El enfoque de la investigación fue de tipo mixto utilizando técnicas de muestreo que arrojaron información tanto cualitativa como cuantitativa, por la importancia de conocer la perspectiva subjetiva y objetiva de los sujetos a investigar, con un diseño 
observacional, el cual se define como aquel cuyo objetivo es la observación y registrando todos los elementos que nos ayuden a la recopilación de información, sin intervenir en el contexto de los sujetos investigados (Hernández-Sampieri, R, Mendoza Torres, 2018).

\section{Resultados}

Se adquirió el consentimiento de los participantes para la utilización y publicación de estos datos, obteniendo los resultados siguientes:

Figura No. 1. ¿El contenido del programa del curso de Psicología fue coherente con lo que recibió durante la clase?

\section{Contenido curricular}

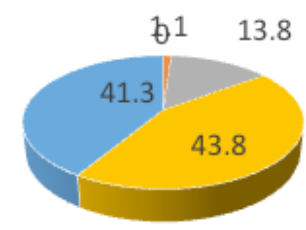

- Nunca " Pocas veces " Algunas veces " Varias veces "Siempre

Fuente: propia.

Se percibe una coherencia existente entre el currículum formal (lo descrito en la programación didáctica) y el currículum real (lo que se imparte dentro del salón de clases), pero no todos los docentes utilizan la misma metodología didáctica, por lo que tanto estudiantes como egresados, perciben que se requiere unificar la metodología que utilizan los profesores para impartir sus clases y de esta forma, todos los estudiantes tendrán la misma oportunidad de desarrollar las competencias que requerirán en su práctica médica.

En la siguiente gráfica, se describe la percepción de estudiantes, egresados y docentes en cuanto a las perspectivas y paradigmas educativos 
Figura No. 2. ¿Qué descripción se adapta más a las características del currículum de la unidad didáctica de psicología?

\section{Perspectivas educativas}

43.8

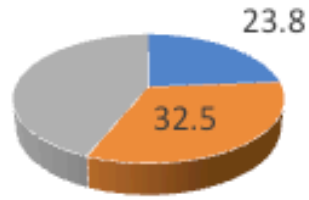

- Currículum como producto, evaluación por objetivos y niveles, profesores como mediadores

- Currículum práctico, evaluación constante, profesor como educador y evaluador

- Currículum con una relación dialéctica (desarrollo científico, profesional y social) evaluación con reflexión crítica y análisis continuo, profesor como una persona que comprende los intereses del estudiante

Fuente: propia.

Figura No. 3. ¿Qué descripción se adapta más a las características del currículum de la unidad didáctica de psicología?

\section{Perspectivas educativas}

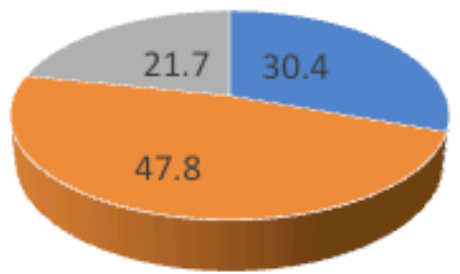

- Currículum como producto, evaluación por objetivos y niveles, profesores como mediadores

- Currículum práctico, evaluación constante, profesor como educador y evaluador

- Currículum con una relación dialéctica (desarrollo científico, profesional y social) evaluación con reflexión crítica y análisis continuo, profesor como una persona que comprende los intereses del estudiante

Fuente: propia. 
Docentes: Grupo focal "Nuestra unidad nos lo permite, el poder estar dentro de los tres paradigmas y perspectivas, por el tipo de profesores y el tipo de unidad didáctica que somos que es psicología y que estamos enfocados a lo que es el humanismo, además de la diversidad de actividades evaluativas" Informante No. 9

En cuanto a las perspectivas y paradigmas educativas, existe una discrepancia entre los tres sujetos involucrados en la investigación, catalogando los estudiantes el currículum de psicología, con una perspectiva crítica o de evaluación para la acción y un paradigma crítico. Los egresados por otra parte ven el currículum de psicología con una perspectiva hermenéutica o de evaluación para la comprensión y un paradigma cuantitativo. Y para finalizar los docentes por medio de un grupo focal, describen la utilización de las tres perspectivas y paradigmas dependiendo el modelo de evaluación que utilizan, el rol docente dentro del salón de clases y el desempeño de los estudiantes.

Existe una discrepancia entre los tres sujetos inmersos en la investigación, ya que los estudiantes tienen la percepción de que la metodología utilizada en el curso de psicología les permitió, comprender los contenidos de forma reflexiva, mientras que los egresados, lo perciben a un nivel de comprensión teórico, más no en su aplicación. El personal docente, percibe que si se cumple con el perfil de egreso del médico y cirujano en un estudio biopsicosocial del individuo a lo largo del año. Es importante también, brindarle la orientación adecuada al estudiante, cuando identifique aspectos que requieran atención psicoterapéutica y donde adquirirla

El curso de psicología se recibe en una temporalidad muy temprana en la carrera de la carrera de médico y cirujano y el mismo no vuelve a repetirse a lo largo del currículum siendo este un instrumento que comunica los principios y propósitos educativos, que promueve una discusión crítica que nos ayuda a realizar una práctica adecuada, de los conocimientos obtenidos (Stenhouse, 2003). Se requiere más acompañamiento en temas psicológicos o que le den seguimiento en años posteriores. Además, es importante la capacitación docente en psicología clínica y este enfoque al contenido del curso. 


\section{Discusión}

Es necesaria la implementación de programas de capacitación continua al personal docente, en el modelo por competencias que se está utilizando en la facultad de ciencias médicas, orientándolos en el aprendizaje enfocado en responder a las necesidades del contexto y así lograr un aprendizaje significativo y autónomo, desde un enfoque biopsicosocial.

Definir las perspectivas y paradigmas desde el claustro docente de la Unidad Didáctica de Psicología, en coherencia con el modelo educativo por competencias que se utiliza en la facultad de ciencias médicas y se logre unificar, desde la perspectiva docente, hasta la aplicación con los estudiantes en el proceso de enseñanza - aprendizaje

Orientar al docente de la unidad didáctica de psicología a la aplicabilidad de los contenidos del curso, para que los estudiantes desarrollen niveles de desempeño, donde logren un aprendizaje autónomo y significativo, a la vez, desarrollen habilidades cognitivas, el dominio de técnicas y métodos generando en él, un cambio actitudinal y que desarrolle destrezas sociales

Siendo la salud mental un elemento tan importante en la formación de la personalidad del individuo y de la atención médico paciente, es importante incluir cursos que le den seguimiento, al curso de psicología que se imparte en el primer año de la carrera de medicina, o contenidos que refuercen en años posteriores la importancia de la salud mental. Ya que la falta de la misma afecta la coherencia entre los procesos de pensamiento, afectividad y la capacidad de adaptación de los estudiantes frente a la vida. También ayuda a determinar cómo maneja el estrés, las relaciones con los demás y toma de decisiones, aspectos importantes en la práctica médica

Realizar periódicamente evaluaciones en el currículo de la unidad didáctica de psicología, y establecer un plan de mejora, con la finalidad de aminorar las brechas tomando en cuenta las áreas de mejora encontradas y así coadyuvar a la calidad educativa. A la vez, es importante brindar seguimiento a los egresados y así tener 
un panorama de la efectividad del perfil de egreso descrito en el currículum de la carrera de médico y cirujano de la Universidad de San Carlos de Guatemala.

\section{Agradecimientos}

Agradezco al Maestro Marco Antonio Castillo, quien fue mi docente en los cursos de seminario de investigación, así como también asesor en la presente investigación, y a la Doctora Patricia Luz Mazariegos Romero, por sus conocimientos impartidos como docente y también revisora en dicho proceso.

\section{Referencias}

Casanova, M. (2009). Diseño y desarrollo curricula innovación educativa. Madrid: La Muralla.

Casarini, M. (1999). Teoría y diseño curricular. México: Trillas.

Hernández-Sampieri, R. \& Mendoza, C (2018). Metodología de la investigación. Las rutas cuantitativa, cualitativa y mixta, Ciudad de México, México: Editorial Mc Graw Hill Education, Año de edición: 2018

Stenhouse, L. (2003). Investigación y desarrollo del currículum. Madrid: Morata 


\section{Sobre la autora}

\section{Vilma Estela García Marroquín}

Es licenciada en psicología de la Universidad de San Carlos de Guatemala, con técnico en orientación vocacional y laboral y un diplomado en gestión de recursos humanos de la Escuela de Psicología, actualmente docente de la unidad didáctica de psicología de la facultad de ciencias médicas -USAC.

Copyright (c) Vilma Estela García Marroquín

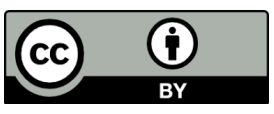

Este texto está protegido por una licencia CreativeCommons 4.0.

Usted es libre para compartir, copiar y redistribuir el material en cualquier medio o formato y adaptar el documento, remezclar, transformar y crear a partir del material para cualquier propósito, incluso comercialmente, siempre que cumpla la condición de atribución: usted debe reconocer el crédito de una obra de manera adecuada, proporcionar un enlace a la licencia, e indicar si se han realizado cambios. Puede hacerlo en cualquier forma razonable, pero no de forma tal que sugiera que tiene el apoyo del licenciante o lo recibe por el uso que hace. 